\title{
Optical Rotor-Blade Deformation Measurements using a Rotating Camera
}

\author{
Fritz Boden ${ }^{1}$, Bolesław Stasicki ${ }^{1}, K_{\text {rzysztof Ludwikowski }}{ }^{2}$ \\ ${ }^{1}$ German Aerospace Center, Institute of Aerodynamics and Flow Technology, Göttingen, Germany \\ ${ }^{2}$ HARDsoft Microprocessor Systems, Kraków, Poland \\ fritz.boden@dlr.de
}

\begin{abstract}
:
For the design of rotors for helicopters or wind turbines, the knowledge of movements and deformations of the rotor blades is important. Measuring these parameters in the rotating system is difficult, because the number of sensors is limited due to their impact on the aerodynamics and the modification of the structure. Strain gauge measurements furthermore can be affected e.g. by the sensor location or temperature effects. To avoid those problems and in addition enable direct shape measurements, optical methods have been applied to rotor deformation measurements in the past. These attempts have been done out of the rotating frame observing either only small rotors or the blade passing the field of view. DLR and Hardsoft developed a rotating 3D imaging system for helicopter rotors and performed tests on the whirltower of Airbus Helicopters in Donauwörth (GER). The system is mounted on the hub and co-rotates with the rotor. It is able to record images of the whole blade at each azimuth angle. Those images later are processed with an Image Pattern Correlation Technique (IPCT) software tool and deliver the 3D surface shape and location of the observed blade. The paper briefly describes the rotating camera system, the non-intrusive deformation measurement method IPCT and the measuring setup, as well as the obtained data and a discussion of the measurement results.
\end{abstract}

Key words: IPCT, rotating camera, blade deformation, optical measurements, stereoscopic imaging

\section{Introduction}

For the proper design and the secure operation of rotor systems the occurring blade deformations and movements are of great importance. Especially for blades with a high aspect ratio like on helicopter main rotors or wind turbines the knowledge about the flapping motions and the blades behavior at the natural frequencies is crucial.

Usually for modal analysis and testing accelerometers and strain gauges are applied. For measurements on the rotating blades commonly strain gauges are preferred, because any sensor placed on the surface should affect the aerodynamic properties as little as possible and thus the sensor size should be very small. The measured strains can be twice integrated in order to obtain the blade twist and bending. To perform this calculation insufficient accurate way, a large number of measuring stations is required. If the expected mode shapes are known (e.g. by numerical calculations) and the strain gauge bridges are placed in a clever way the so called strain pattern analysis (SPA)[1] can be applied in order to identify the blade deformation with less sensors. In order to obtain good measurement results, strain gauge measurements should be performed accurately by experienced and skilled staff to minimize the main error sources such as the mounting procedure, transverse sensitivity, temperature effects, humidity effects, strain cycling, fatigue and cable effects [2]. For increasing the accuracy and enabling a little bit larger number of measurement locations fiber Bragg grating (FBG) sensors can be used for a strain-based modal analysis [3]. But also those sensors are effected by the ambient conditions and require an accurate installation by experienced staff.

To avoid the problems mentioned above and in addition enable direct shape measurements contactless optical methods have still been applied to rotor and propeller deformation measurements in the past [4]-[8]. These attempts have been done with measurement devices out of the rotating frame observing either only small rotors or the blade passing within the field of view. Former recordings with rotating camera devices on helicopter rotors have been performed just to visualize the flow 
over the blades [9] or to determine the motion of the rotor blade [10].

Based on their experience from the in-flight application of a rotating camera on an aircraft propeller [11], DLR in cooperation with Hardsoft developed a rotating $3 \mathrm{D}$ image acquisition system for helicopter rotors [12] and performed first tests on the whirltower of Airbus Helicopters in Donauwörth, Germany. The system is mounted on the hub and co-rotates with the rotor. It includes four CMOS camera sensors and a complete data acquisition system and is able to record images of the whole blade at each azimuth angle. Later, those images are processed with an IPCT software tool and deliver the continuous 3D surface shape and location of the observed blade. From the comparison of the 3D surfaces for different recordings, the bending and torsion of the blade can be obtained. In what follows this rotating camera system, the IPCT method as well as some example results are given.

\section{Design of the rotating camera system}

In the simplest case a rotating camera system can be realized by a single motion picture camera mounted on the hub co-rotating with the rotor and observing a part of one blade [9]. For qualitative observation of the flow or the blade movements such a system fulfils all requirements, but if quantitative shape and deformation measurements are intended to be performed, such a system should comprise digital imaging sensors best in a stereoscopic arrangement. Such a rotating stereo camera system for 3D blade deformation measurements has been realized by the authors in the past on a propeller aircraft [11].

To observe a complete helicopter main rotor blade, being an object with a high aspect ratio viewed under a flat viewing angle, it was decided to build a double stereo vision system consisting of four lightweight Ximea XiQ MQ042MG-CM global shutter cameras in two stereoscopic arrangements with overlapping fields of view (Fig. 1) in order to enable imaging with sufficient resolution for optical deformation measurements. The cameras each have a resolution of 2,048 pixels $\times 2,048$ pixels with a maximum frame rate of $90 \mathrm{fps}$. The applied lenses as well as the positions of the cameras including the height above the rotor and the base distance between the two cameras of each stereoscopic system iteratively have been chosen in order to obtain an optimum between the size of the field of view and the inaccuracy of the deformation measurements. In Fig. 2 the estimation of the measurement inaccuracy of the final camera setup calculated according to [13] is depicted.

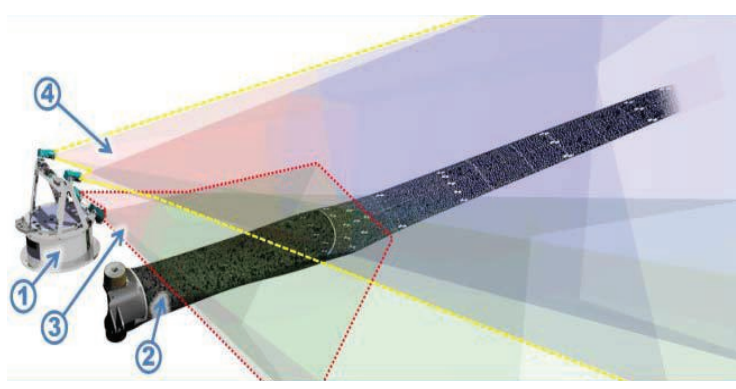

Fig. 1. Fields of view of the rotating camera system for rotor blades $(1$ - rotating camera system, 2 observed rotor blade, 3 - field of view of the lower cameras, 4 - field of view of the upper cameras).

The cameras of the lower stereo-system have C-mount lenses with a focal length of $\mathrm{F}=12 \mathrm{~mm}$, a base distance $\mathrm{B}$ of around $250 \mathrm{~mm}$ and are located approximately $400 \mathrm{~mm}$ above the rotor. They observe the control cuff and the rotor blade attachment with an estimated measurement inaccuracy of 0.4 to $0.55 \mathrm{~mm}$. The cameras of the upper stereo-system are around $600 \mathrm{~mm}$ above the rotor and have lenses with $\mathrm{F}=25 \mathrm{~mm}$, a base distance $B=280 \mathrm{~mm}$. They observe the complete blade from the attachment to the tip. The estimated measurement inaccuracy goes from $0.2 \mathrm{~mm}$ (in the area overlapping with the lower camera system) up to $1.4 \mathrm{~mm}$ at the blade tip. Because all devices located in the rotating system of a helicopter are exposed to enormous centrifugal forces and vibrations, the cameras as well as the required recording hardware need to be mounted on the rotor hub in a compact and rigid way. Furthermore it should be enabled to perform a fine adjustment of the camera viewing angles in-situ. The final design of the rotating camera system is shown in Fig. 3. The system has a maximum diameter of $340 \mathrm{~mm}$ and is approximately $370 \mathrm{~mm}$ high. The lowest part is an interchangeable connecting flange (item 1 in Fig. 3) enabling the mounting of the rotating camera system on different rotor hub geometries. The big aluminum cylinder (item 2 in Fig. 3) contains all electronics required for the image acquisition.

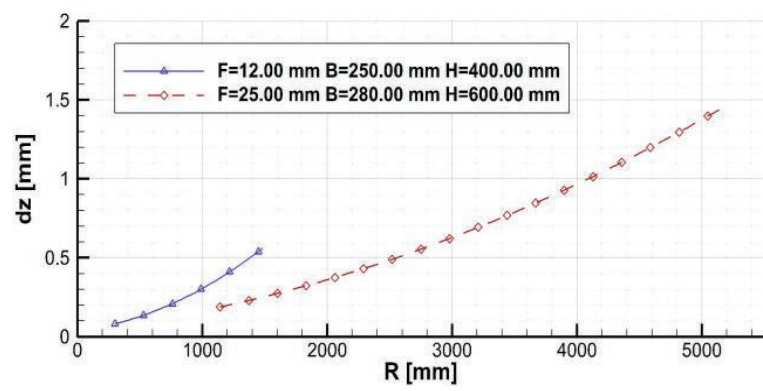

Fig. 2. Estimated measurement inaccuracy of the designed rotating camera system according to [13] (dz - inaccuracy, $R$ - rotor radius, $F$ - focal length of the cameras, $B$ - base distance between cameras, $H$ - height of camera system above rotor). 


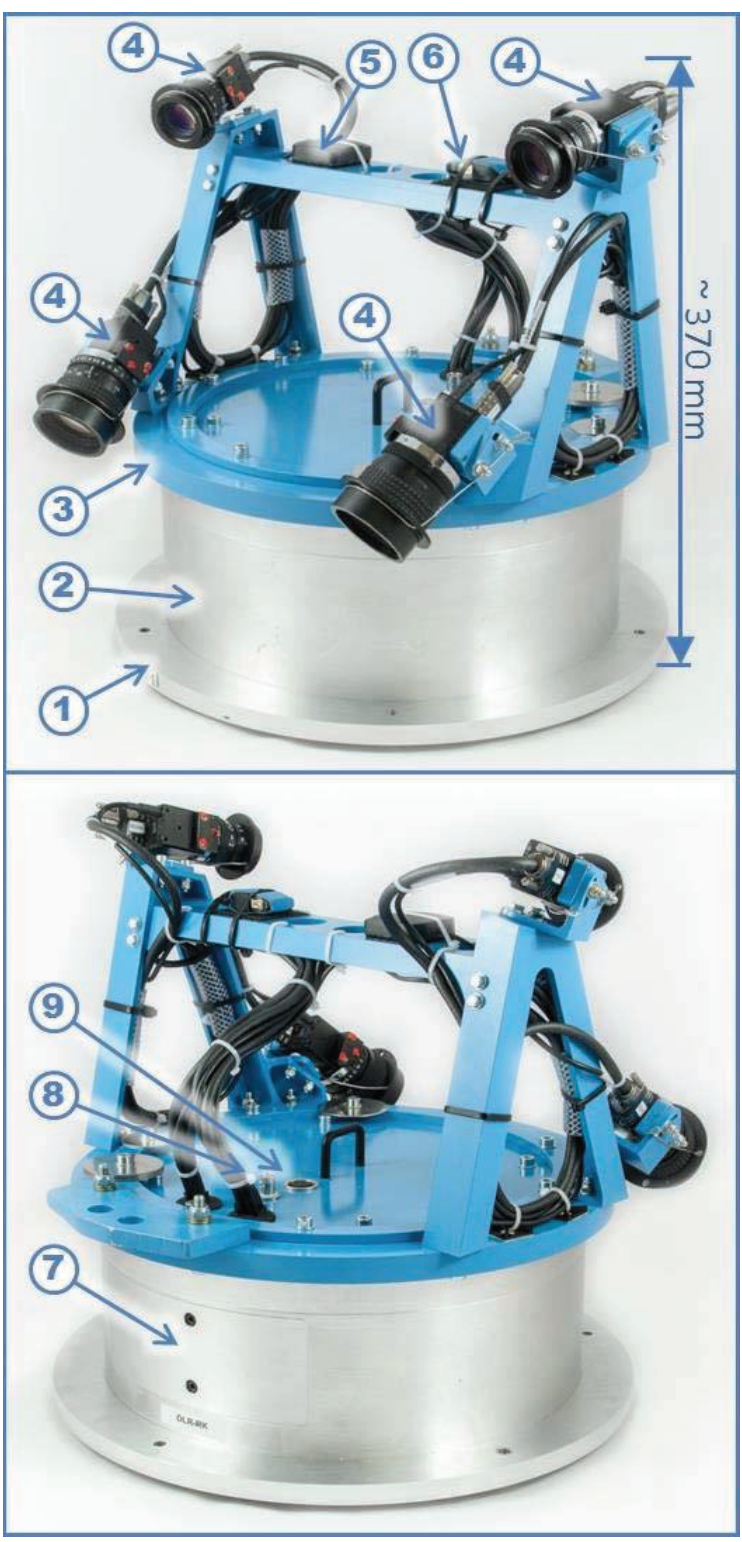

Fig. 3. The rotating camera device (1-interchangeable mounting flange, 2-electronics enclosure, 3-adjustable camera mounting support, 4 - cameras including optics, 5-GPS antenna, 6WLAN antenna, 7-service connector compartment, 8-trigger connector, 9-connector for external power supply).

one embedded PC as control computer, a multichannel contains all electronics required for the image acquisition - one embedded PC as control computer, a multichannel phase shifter card [14] for accurate triggering at multiple phase angles during each revolution, four removable solid state drives (SSD) for the operating system and storing the data as well as two battery packs (optional for autonomous operation). The printed circuit boards (PCB) are mounted in single stages inside the cylinder prevented against rotational forces and vibrations like in a corset. On top of the cylinder the lightweight rigid camera support (item 3 in Fig. 3) is seated.

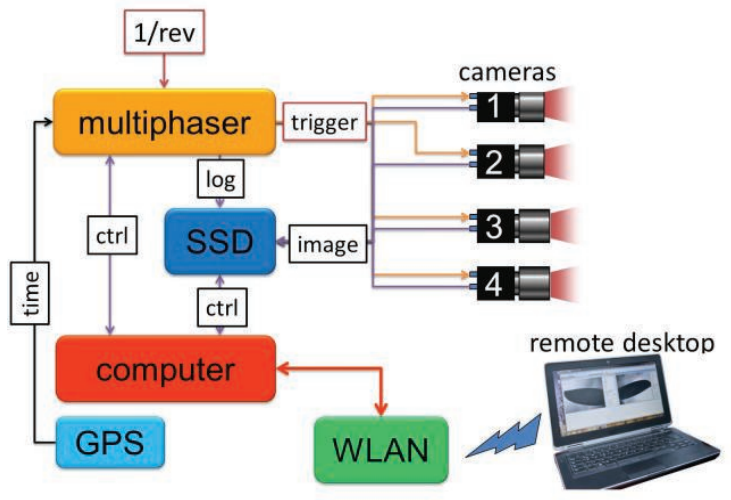

Fig. 4. Schematic sketch of the rotating camera system.

When releasing the clamping mechanism the complete support can be rotated around the vertical axis enabling the observation of different blades without dismantling the rotating camera from the rotor hub. The four cameras (item 4 in Fig. 3) are affixed at this camera support on folded sheets allowing a fine adjustment of \pm 20 degree around the vertical axis and the line of sight of each single camera. Antennas for GPS (item 5 in Fig. 3) and a WLAN (item 6 in Fig. 3) are used for time synchronization and remote control, respectively. Connectors for an external trigger signal (item 8 in Fig. 3) and external power supply (item 9 in Fig. 3) are situated on the detachable cover of the aluminum cylinder. Behind a cover plate (item 7 in Fig. 3) at the side of the cylinder additional connectors for a monitor and USB devices are located for the purpose of a test and general setup of the system on ground. The working principle of the rotating camera system schematically is shown in the flow chart in Fig. 4. A TTL signal, e.g. an index pulse occurring once per revolution $(1 / \mathrm{rev})$, is provided to the multichannel phase shifter[14] (based of the previously developed digital phase shifter [15]). This "multiphaser" uses the $1 / \mathrm{rev}$ signal to calculate the present rotor speed and exactly triggers the four cameras to take images at the rotor azimuth angles programmed with the computer. The images are directly stored on the removable SSDs together with a log file containing the image number, the phase or azimuth angle, the rotational frequency, the GPS time and position as well as the camera settings. The recording of such single images in combination with the log file eases the later processing of the image data compared to the recording of video data where usually only fix frame rates are possible and the access to dedicated single frames is difficult. Furthermore these log files ease the synchronization of the image data with other events and measurements happening in parallel to the image recording. 


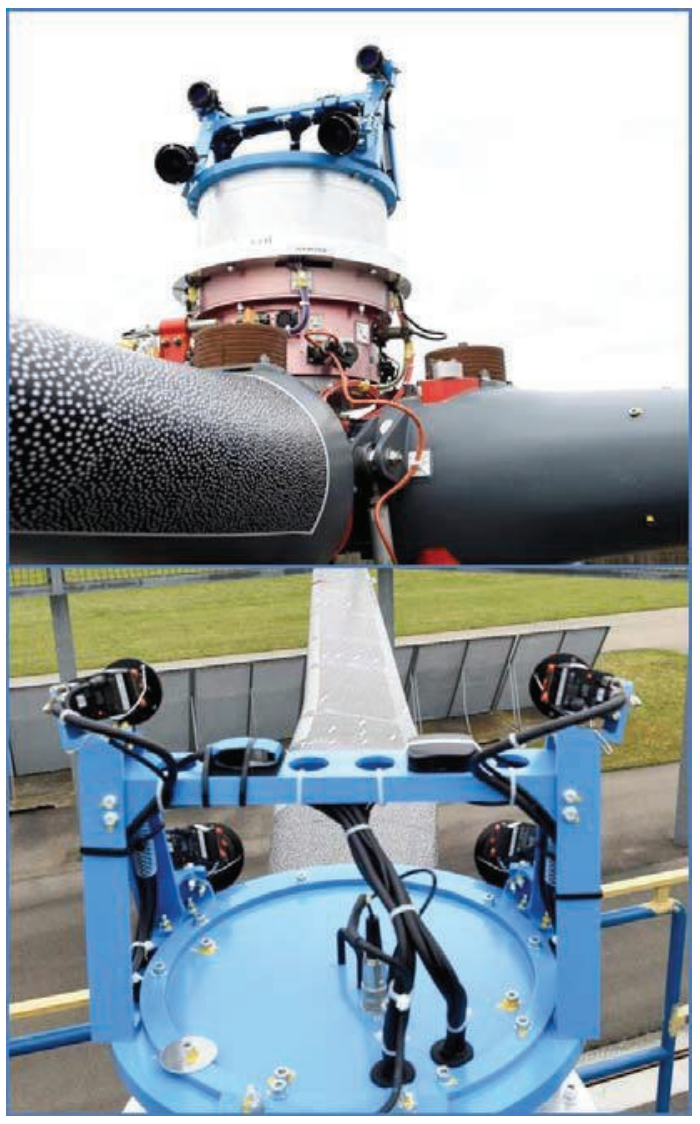

Fig. 5. The rotating camera system mounted on the experimental rotor (left-view on the mounted camera system, right-view along the patterned blade).

To allow a remote control of the camera system, e.g. for starting recording sequences, changing the camera exposure settings, or checking recorded images, a laptop computer is linked to the rotating camera system using a WLAN connection and a remote desktop application. After the performed test, the SSD with the gathered image data can easily be removed from the rotating camera device and used for offline data processing

\section{An example measurement campaign}

During a measurement campaign within the german national project CHARME (CHallenge in AeRothermoMEchanics) the developed rotating camera system was tested on the Airbus Helicopters whirltower in Donauwörth (Germany). A standard rotor measurement program was performed including static deformation as well as vibration measurements. Besides the measurement by means of the rotating camera additional deformation measurement systems have been applied during the test, such as an instrumentation of the blade with strain gauges and another optical measurement system standing on ground. In addition transition measurements by means of Infrared-Thermography have been performed.

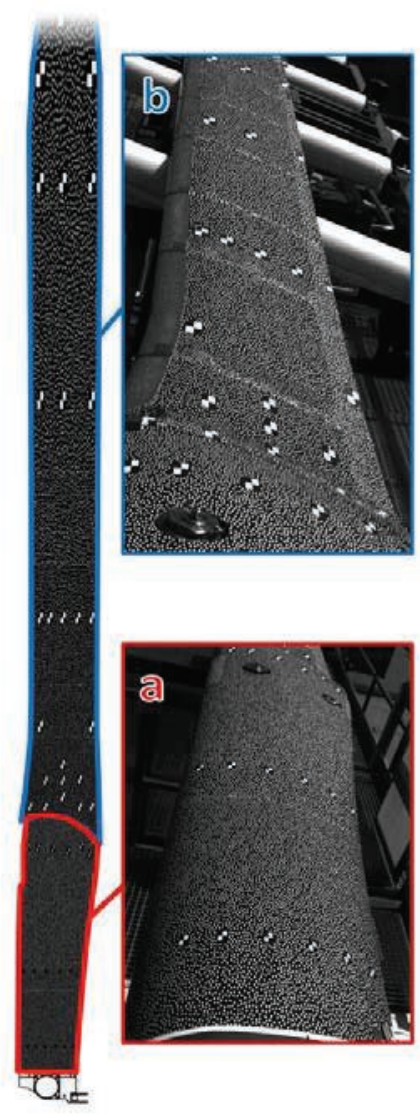

Fig. 6. Overview on the designed IPCT pattern for the control cuff and rotor blade (a - sticker with printed pattern on control cuff, $b$ - pattern painted directly on the blade surface).

For the optical deformation measurements using the image pattern correlation technique (IPCT) the rotating camera was mounted on the rotor hub (see Fig. 5) observing one of the rotor blades covered by a stochastic dot pattern. Two types of pattern application method have been tested - a printed pattern on a sticker on the control cuff and the pattern painted on the blade using a paint mask. The advantages of the sticker method are the high quality of the printed pattern and the fast and easy application and removal of the sticker. As a drawback the sticker can be peeled off by the flow especially at locations with high pressure gradients. Therefore in those regions the pattern should be directly painted on the blade surface. For a proper painting a paint mask manufactured with a cutting plotter is recommended. The design of the pattern has been done with the help of the known 3D CAD geometry and a digital mock-up tool (DMU) [11], [16]. In Fig. 6 an overview of the pattern as well as the two different regions (sticker and paint) are given. It is clearly visible that the sticker provides a much better reproduction of the pattern design - on the painted part inhomogeneities (e.g. due to the covering material on the strain gauge instrumentation) 
are also seen in the pattern whilst the sticker covers that different backgrounds. Taking a look to the pattern itself, it can be seen, that beside the stochastically distributed dots additional checker board markers are included. Those markers serve as reference points to merge the results of the lower and upper camera systems, to correct unwanted camera movements and to get initial information about corresponding areas between the two cameras of each stereoscopic system. Furthermore the designed pattern and the markers are increasingly stretched towards the blade tip. This ensures a similar size of the image of all parts of the pattern on the camera sensors, as it can be seen on the camera images in Fig. 6 and Fig. 7. Prior to the mounting on the rotor the rotating camera system had been balanced in order to avoid unwanted vibrations in the rotor system. After a successful first functional test with running rotor the real measurement campaign started. Before each run a 3D camera calibration has been performed by placing a checker board calibration target in front of the cameras and record images whilst moving the target along the blade. The recorded images are later used for the determination of the intrinsic and extrinsic camera parameters [17]. Fig. 7 shows a set of sample recordings taken during the measurement campaign - on the left side the images recorded by camera 1 and camera 2 (upper stereoscopic system) and on the right side the images recorded by camera 3 and camera 4 (lower stereoscopic system). The high resolution images show sufficient contrast and sharpness of the IPCT pattern and the markers. The background behind the blade is naturally blurred due to the high rotational speed. The overlapping area between both stereoscopic systems located around the blade attachment is well represented in all images.

\section{Image processing by means of IPCT and example results}

After the test, the recorded stereoscopic image pairs are processed by means of the image pattern correlation technique (IPCT) using a software tool developed by DLR. Fig. 8 delivers a short overview on the main steps from stereoscopic images to measured 3D surfaces. First of all the calibration images (Fig. 8-1) are evaluated in order to deduce the transformation matrix between image and world co-ordinates. Next a marker detection algorithm is applied to the measurement images (Fig. 8-2) to find the image coordinates of corresponding markers (Fig. 8-3). If those image co-ordinates are triangulated utilizing the $3 \mathrm{D}$ calibration, the $3 \mathrm{D}$ positions of the markers can be obtained as a first result.

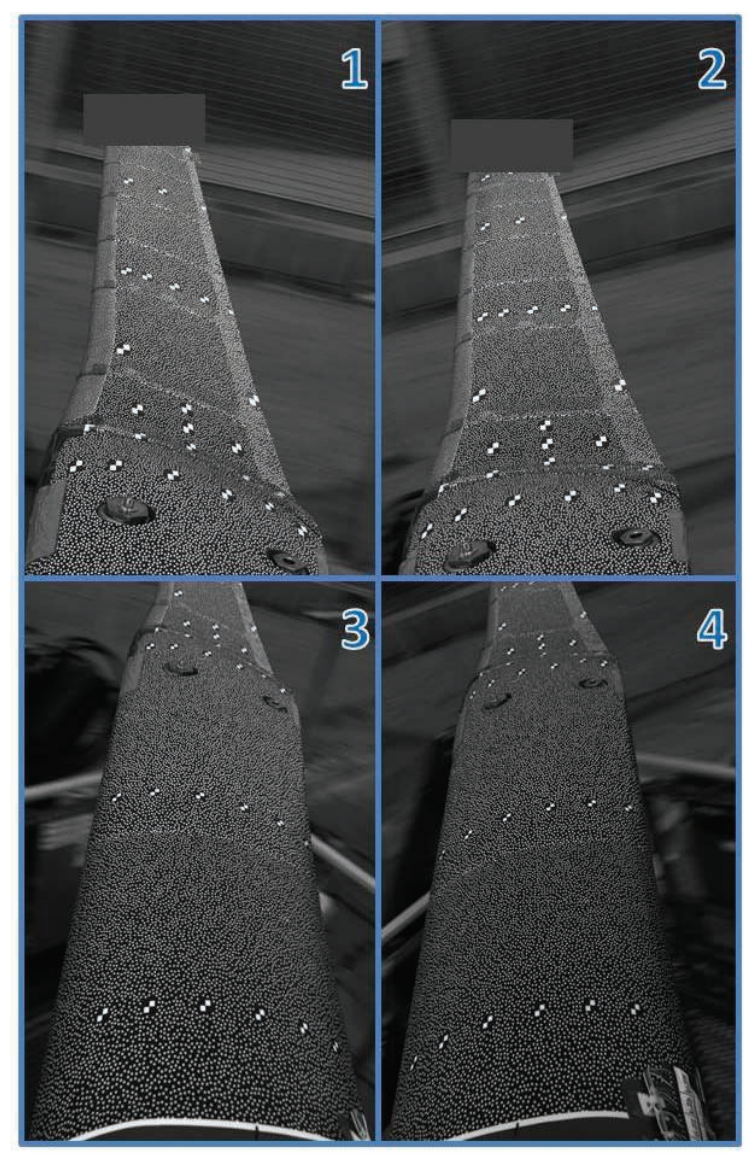

Fig. 7. Example recordings taking at full rotor speed $(1+2=$ upper stereoscopic system (camera 1 and camera 2), $3+4=$ lower stereoscopic camera system (camera 3 and camera 4).

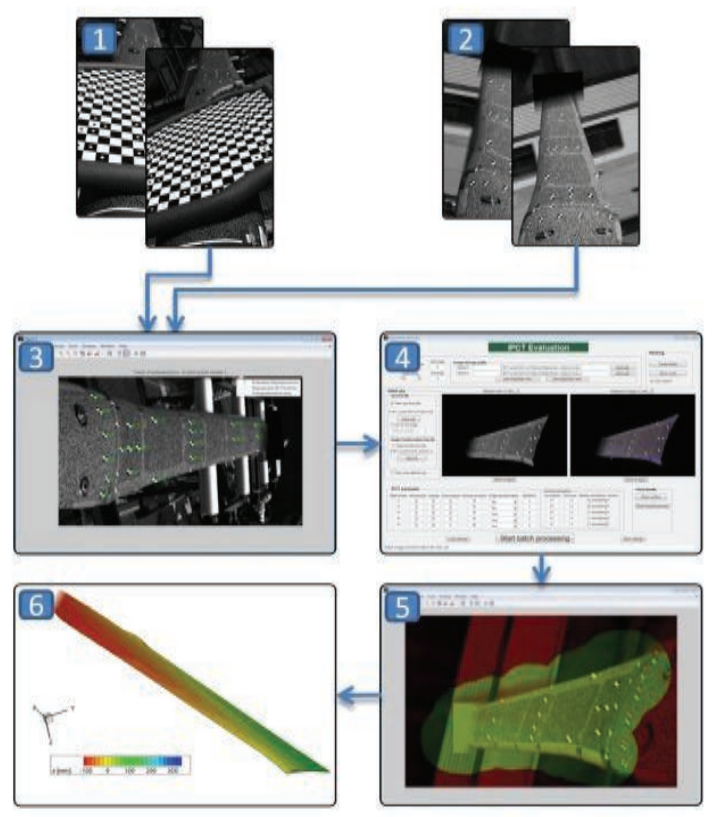

Fig. 8. Scheme of IPCT processing ( 1 - images for stereo camera calibration, 2 -stereoscopic measurement images, 3 -Marker detection procedure, 4 - image pattern correlation, 5 - overlay of successfully deformed images, 6 -triangulated resulting surface). 


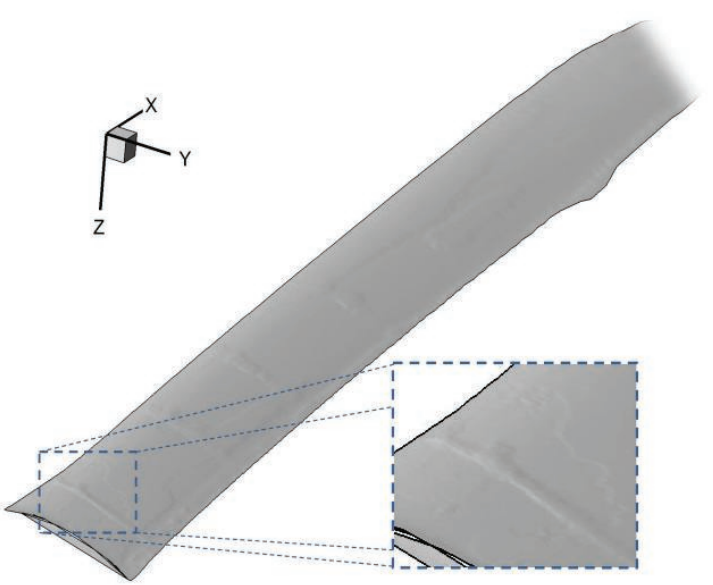

Fig. 9. Example surface of the rotor blade obtained from IPCT processing with zoomed detail (remark: the blade tip is faded out due to confidentiality reasons).

For the following image pattern correlation (Fig. 8-4) the image co-ordinates of the markers are used to get start values for an initial image deformation. By means of an iterative cross correlation more and more corresponding points are identified in the two stereoscopic images and the image deformation is improved until both images are matching as shown in Fig. 8-5 by an overlay image. In the last step the corresponding points are triangulated using the 3D calibration resulting in the reconstruction of the measured 3D surface of the blade (Fig. 86). In the references [13], [16], [17] further information about the IPCT processing can be found.

An example surface obtained from the measurement images by means of IPCT is depicted in Fig. 9. The surface is smooth and in agreement with the shape of the investigated blade. Like shown in the zoomed box even small details on the blade surface can be reconstructed - in that case the raise due to the covering material of the strain gauge installation.

If the IPCT processing is performed for different measurement points, the movement and the deformation of the observed blade can be determined. Fig. 10 shows the surfaces for a measurement sequence with nine different collective pitch settings. For each collective pitch setting ten surfaces are taken. The small graphs on the right side show lines extracted in spanwise and chordwise direction identifying the blade bending and the local change of the pitch angle as well as the local heave. As expected the increasing heave with increasing blade pitch angle and increasing spanwise location is clearly visible. Furthermore the growing lag of the blade for higher pitch angles can be seen.

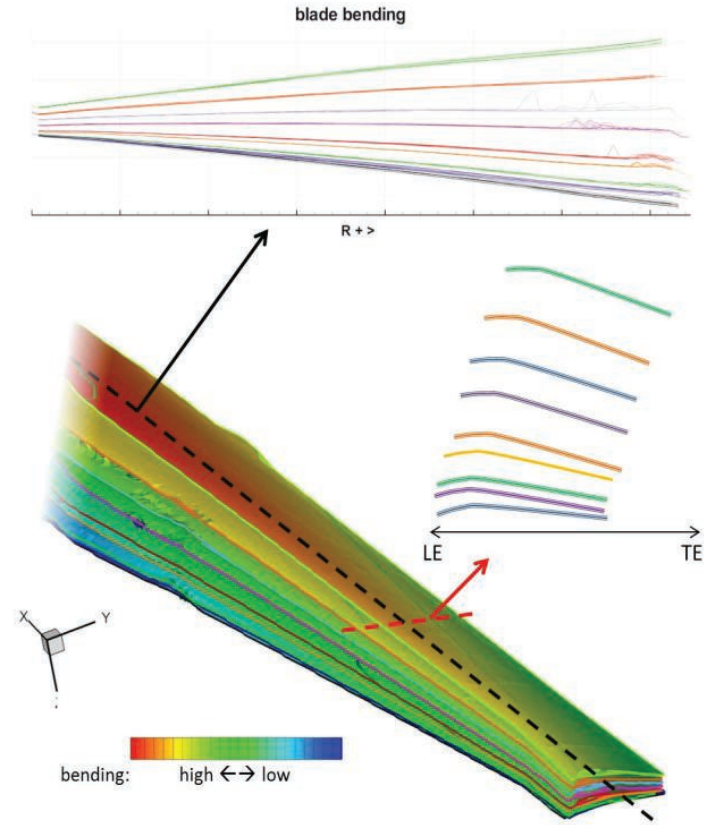

Fig. 10 Example surfaces (left) for different measurement points including spanwise extracted blade bending curves (top right) and chord wise extracted profiles(bottom right) (remark: the blade tips are faded out and the graphs are not to scale due to confidentiality reasons).

Beside the big displacements between the nine sets of measurement points with different pitch settings, a small flapping motion can be observed for the ten lines with the same pitch setting.

\section{Conclusion}

Based on the experiences in optical deformation measurements in rotor systems gained from the application of a rotating camera system mounted on an aircraft propeller a remote controlled rotating camera system with four imaging sensors has been successfully applied to optical rotor blade deformation measurements. The system was able to record the complete main rotor blade (including control cuff, blade attachment and the overall blade) during its full revolution under real operation conditions.

During the measurement campaign the rotor system was recorded under different loading conditions including different rotor speeds, different collective and cyclic pitch settings as well as excited natural frequencies of the rotor resulting in a huge amount of valuable image data processable by means of IPCT. The preliminary processing of some of these images demonstrated the feasibility of the application of IPCT measurements with a rotating camera system.

Even without the IPCT processing the recorded images deliver a novel and valuable insight in 
the behavior of the running rotor system that can not directly be observed with classical methods or with camera systems on the ground. The recorded images can also be used in order to check if movements observed from the non-rotating frame are due to e.g. trigger jitter or if they are real movements of the rotor system.

Compared to a classical strain gauge setup, the instrumentation for optical deformations measurements with the rotating camera system was relatively easy - only a pattern had to be applied on the blade and the camera system had to be mounted on the hub. In addition (if a pattern sticker or washable paint is applied) the installation can be removed after the test and the investigated blade can then be used like a normal production blade.

As the system is co-rotating with the blade the camera exposure times can be much longer than for cameras watching the passing blade from the fix frame. Thus the background illumination (e.g. the sunlight) is sufficient for image recording i.e. no additional strobe light is required. Nevertheless reflections of the sunlight on the investigated surface should be avoided, because they disturb the measurements.

Like mentioned above, the images taken during the test and the first IPCT processing of the data look very promising and deliver a first proof of concept. In the next step all image data has to be processed with the IPCT and shall be compared to the strain gauge measurements, the parallel applied optical measurement from the non-rotating frame, as well as the existing numerical simulations for the blade behavior. In case of the very probable successful comparison the authors are looking forward to test the helicopter camera in flight in the near future.

Besides the in-flight application of the imaging technique, the method is applicable for other fast rotating objects like wind turbine rotors, ship propellers or wheels, either in combination with deformation measurement techniques like the IPCT or with other measurement techniques for optical flow or pressure measurements.

\section{Acknowledgement}

The performed measurement campaign was part of the project CHARME (funding code 20H1501A) funded by the German Federal Ministry for Education and Research (BMBF) where DLR has been subcontracted by Airbus Helicopters to perform measurements with a rotating camera.
The authors furthermore would like to thank the personnel of Airbus Helicopters for the realization of the whirltower test at their premises in Donauwörth.

\section{References}

[1] N. Tourjansky, E. Szechenyi, The measurement of blade deflections - A new implementation of the strain pattern analysis, ONERA TP - 92-127 (1992).

[2] K. Papadopoulos, E. Morfiadakis, T.P. Philippidis, D.J. Lekou, Assessment of the strain gauge technique for measurement of wind turbine blade loads, Wind Energy 3(1), 35-65 (2000)

[3] F.L.M. dos Santos, B. Peeters, B., On the use of strain sensor technologies for strain modal analysis: Case studies in aeronautical applications, Review of Scientific Instruments 87 (10), 102506 (2016); doi: 10.1063/1.4965814

[4] J. Sicard, J. Sirohi, Measurement of the deformation of an extremely flexible rotor blade using digital image correlation, Measurement Science and Technology 24 (6), 065203 (2013); doi:10.1088/0957-0233/24/6/065203

[5] G.A. Fleming, S.A. Gorton, Measurement of rotorcraft blade deformation using projection moiré interferometry, Shock and Vibration 7(3), 149\{165 (2000);

[6] F. Boden, C. Maucher, Blade deformation measurements with IPCT on an EC 135 helicopter rotor, Advanced In-Flight Measurement Techniques, ch. 13, 195-213, Springer Verlag, Heidelberg New York Dordrecht London (2013); doi: 10.1007/978-3-642-34738-2_13

[7] C. Lanari, B. Stasicki, F. Boden, A. Torres, Image based propeller deformation measurements on the Piaggio P 180, Advanced In-Flight Measurement Techniques, ch. 9, 133153, Springer Verlag, Heidelberg New York Dordrecht London (2013); doi: 10.1007/978-3642-34738-2_9

[8] P. Beaumier, B. van der Wall, K. Pengel, C. Kessler, M. Gervais, Y. Delrieux, J.-F. Hirsch, P. Crozier, From ERATO basic research to the Blue $\mathrm{Edge}^{\mathrm{TM}}$ rotor blade: an example of virtual engineering?. Rotorcraft Virtual Engineering Conference (November 2016). LIVERPOOL, United Kingdom. HAL Id: hal-01413109.

[9] F.B. Gustafson, G.C. Myers, Stalling of helicopter blades, NACA TN No. 1083 (1946).

[10] E. Zappa, L. Trainelli, R. Liu, A. Rolando, F. Rossi, P. Cordisco, E. Vigoni, M. Redaelli, Real time contactless sensor for helicopter blade angle measurement, Metrology for Aerospace, IEEE pages 239-244 (2016); doi:

10.1109/MetroAeroSpace.2016.7573219

[11] F. Boden, B. Stasicki, M. Szypuła, P. Ružička, Z. Tvrdik, K. Ludwikowski, In-flight measurements of propeller blade deformation on a VUT100 cobra aeroplane using a co-rotating camera system, Measurement Science and Technology 27.7, 
74013-74025 (2016); doi:10.1088/09570233/27/7/074013

[12] Involved DLR-patents: DE4309353.1, DE195 44 642, DE10 2011001 268. B4, DE 102014117 655.3.

[13] T. Weikert, Basic optics and camera modelling, AIM ${ }^{2}$ Advanced Flight Testing WorkshopHANDBOOK of ADVANCED IN-FLIGHT MEASUREMENT TECHNIQUES, ch. 3, 37-48, BoD - Books on Demand, Norderstedt (2013);

[14] F. Boden, B. Stasicki, K. Ludwikowski, Multikanalphasenschieber, Patent DE 102014 117655 (2014).

[15] B. Stasicki, G.E.A. Meier, Digital phase shift method, Patent DE 19544642 (1997).

[16] T. Kirmse, F. Boden, H. Jentink, Image pattern correlation technique (IPCT), AIM ${ }^{2}$ Advanced Flight Testing Workshop - HANDBOOK of ADVANCED IN-FLIGHT MEASUREMENT TECHNIQUES, ch. 6, 63-85, BoD - Books on Demand, Norderstedt (2013).

[17] T. Kirmse, Recalibration of a stereoscopic camera system for in-flight wing deformation measurements, Measurement Science and Technology 27(5), 054001 (2016); doi:10.1088/0957-0233/27/5/054001 